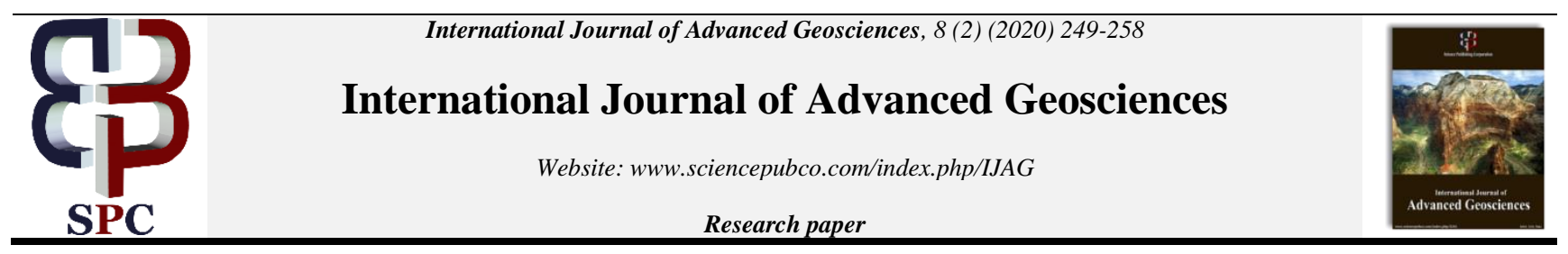

\title{
Assessing groundwater quality using water quality index in semiarid region of Aurangabad district, central India
}

\author{
K.R. Aher ${ }^{1}$, P.L. Salve ${ }^{2}$, S.G. Gaikwad ${ }^{1}$ \\ ${ }^{1}$ Regional Water Testing Laboratory, Deputy Director, Groundwater Surveys and Development Agency, Marathwada region, Near \\ SAO office, Jyotinagar, Darga road, Aurangabad-431005, India \\ ${ }^{2}$ Deputy Director, Groundwater Surveys and Development Agency, Konkan Region, Konkan Bhavan, CBD Belapur, Navi Mumbai, \\ Maharashtra-400614, India \\ *Corresponding author E-mail: kailashgis@gmail.com
}

\begin{abstract}
This paper presents a study on the influence of geochemical parameters on groundwater quality in GV-35 watershed of Aurangabad district, Maharashtra, India. Groundwater samples were collected from different locations and their physiochemical quality parameter were analysed. Water quality index (WQI) was determined on the basis of various physical and chemical parameters like pH, electrical conductivity, turbidity, total dissolved solids, total hardness, calcium, magnesium, sulphate, chloride, nitrate, sodium, potassium, carbonate, bicarbonate, fluoride and iron. These parameters were determined for the calculation of water quality index (WQI). During pre-monsoon, $4 \%$ of groundwater samples were excellent, $65 \%$ were good; $26 \%$ were poor; and $4 \%$ were unsuitable for domestic suitability, whereas in post-monsoon, $65 \%$ of water samples were good; $26 \%$ were poor; $4 \%$ were very poor and $4 \%$ were unsuitable for domestic suitability
\end{abstract}

Keywords: GV-35 Watershed; Water Quality Index; Domestic Suitability; Aurangabad; India.

\section{Introduction}

Ground water is one of the main sources of water requirement of people in India as well as other parts of the world. Pollution of water has been reported to cause $80 \%$ of human diseases and $30 \%$ of infant mortality. It is, therefore, important to monitor the quality of ground water pollution of various parts of our country (Singh \& Parwana, 1992). Anthropogenic activities can alter the relative contributions of the natural causes and also introduce the effects of pollution (Whittemore et al,1989; Salve and Aher, 2016). Groundwater is the major source of water for domestic, agricultural and industrial purposes in many countries. India accounts for $2.2 \%$ of the global land and $4 \%$ of the world water resources and has $16 \%$ of the world's population. It is estimated that approximately one third of the world's population use groundwater for drinking (Nickson et al, 2005). Therefore, water quality issues and its management options need to be given greater attention in developing countries. Intensive agricultural activities have increased the demand on groundwater resources in India. Water quality is influenced by natural and anthropogenic effects including local climate, geology and irrigation practices (Ramesh \& Elango, 2011; Deshpande et al, 2012; Marghade, et al, 2020). Groundwater is the main source that caters the need of inhabitants from rural part for daily activities, especially in a developing country like India (Gaikwad et al, 2018; Kumar et al, 2020; Kate et al, 2020). Water is the essence of life and nearly 165 billion liters of water needed per day in India (Gupta and Misra, 2018). Water quality is an imperative matter directly related to the welfare of the human race. Due to the shortage and contamination of surface water, the dependency on groundwater has been increased within a few decades to meet water requirements for drinking, irrigation, and other uses. The measure of the quality of water used for irrigated agriculture is essential for predicting its long-term usage while attaining enhanced productivity (Bauder et al, 2011; Wagh et al, 2018). All the agricultural practices carried out in the country are majorly dependent on this hidden natural resource (Srivastava and Parimal, 2020; Marghade, 2020; Verma et al, 2020). Specifically, in arid and semiarid provinces, subsurface water is mostly limited due to the scanty precipitation, high evaporation and surface runoff (Camacho Suarez et al, 2015; Kadam et al, 2020). While meeting the demands from various sectors, this precious resource is getting scarce, overexploited and contaminated in many parts of the country (Avvannavar and Shrihari, 2008; Vasanthavigar et al, 2010; 2012; Sharma and Kansal, 2011). Moreover, the ingestion of such polluted groundwater could upsurge the hazards of aquatic infection epidemic (Wu and Sun, 2016; Li et al, 2019). The degrading groundwater quality has become a serious universal issue for sustainable development (Adimalla and $\mathrm{Wu}, 2019$ ). Hence, it is extremely important to understand the hydrogeochemical characteristics of groundwater for sustainable resource development and governance. The quality of ground water is the resultant of all the processes and reactions that have acted on the water from the moment it condensed in the atmosphere to the time it is discharged by a well. Therefore, the quality of ground water varies from place to place, with the depth of water table, and from season to season and is primarily governed by the extent and composition of dissolved solids present in it (Aher, 2012). The variations in major ion chemistry of groundwater lead to identification of geochemical processes that control the groundwater quality. Weathering of minerals within the rocks is an important process, regulating the concentration of dissolved ions in groundwater (Jacks, 1973; Bartarya, 1993; Rao and Rao, 2010). Groundwater is a vital natural resource for the reliable and economic provision of potable water supply in both the urban and rural environment. It thus plays a fundamental role in human well-being as well as that of aquatic and terrestrial ecosystems. In the 
background of preserving this most important natural asset, the water utility management is the key area that managers need to focus upon. Fresh and clean water is of fundamental importance to the survival, protection and development of human needs. The movement of groundwater is controlled by physical and geochemical properties of (i) contaminant (ii) the groundwater and (iii) the geological system through which the contaminated groundwater is flowing. Presence of some ionic contents beyond certain limits may make it unsuitable for irrigation, domestic or industrial uses (Purushtotham, et al, 2011; Aher and Deshpande, 2014). Poor quality of water adversely affects the plant growth and human health (US Salinity Laboratory Staff, 1954). The determination of physicochemical parameter has established role in evaluating the quality of groundwater, which is why these parameters have also been duly focused on. The chemical composition of water has evolved much interest during the last few decades because of several factors. The quality of groundwater is deteriorating mainly due to anthropogenic activity, irrigation return flow, excessive utilization of chemical fertilizers, municipal waste, unhygienic practices, septic tank effluent and landfills leachate (Srinivasmoorthy et. al, 2011; Aher and Dhumal, 2017). The agriculture is a dominant occupation in the study area, and groundwater demand in future will be very high. Along with the concern on the availability of this natural resource, it becomes extremely important to monitor and maintain its compositional value for safeguarding human's health while maintaining the fertility of soil. The Water Quality Index, indicating the water quality in terms of index number, offers a useful representation of overall quality of water for public or for any intended use as well as in the pollution abatement programs and in water quality management (Brown et al, 1972; Chatterjee \& Raziuddin, 2007). Keeping this in view, the present study aims to calculate the water quality index (WQI) in order to assess the suitability water for domestic use.

\section{Study area}

The study area occupied by Bori Basin is located between latitude $19^{\circ} 45^{\prime} 05^{\prime \prime}$ to $20^{\circ} 50^{\prime} 01^{\prime \prime} \mathrm{N}$ and longitude $74^{\circ} 45^{\prime} 00^{\prime \prime}$ to $74^{\circ} 55^{\prime} 05^{\prime \prime}$ E in Vaijapur block of Aurangabad District, Maharashtra, Central India (Fig. 1). Groundwater surveys and development agency nominated this watershed as GV-35 watershed (GSDA,2019). The area covers to extent of about $284.98 \mathrm{~km}^{2}$ and is distributed in 25 villages and includes in the Survey of India topographical maps (46L/16 and 46I/13). The climate of the area is semi-arid, with minimum and maximum mean annual temperatures of $10.3^{\circ} \mathrm{C}$ in winter and $39.8^{\circ} \mathrm{C}$ in summer, respectively. The mean rainfall over a period of 50 years is about 540.85 $\mathrm{mm}$. However, recently, the area has an acute shortage of surface water due to the frequent failures of monsoon. Agriculture is the main source of livelihood (Deshpande and Aher,2012a). Physio-graphically the area is a gradual plain with a gentle slope, which increases towards the north. The direction of the slope decreases towards the south east within the whole study area. The major part of the study is covered by black cotton soil or 'Regur' formed by the weathering of Deccan Trap Basalt. The drainage is dendritic, following the topography. The streams flow towards the south east. Streams are intermittent, except during rainy season (Aher,2017; Aher and Dhumal, 2017). Geologically the whole area covered by Deccan trap of the Late Cretaceous - Palaeogene (68-62 million years age) comprising lava flows of basaltic composition, the Deccan again divided into Lower Ratangarh and upper Ratangarh formations of Sahyadri group. The lower Ratangarh formation occurs in the middle part of the area having thickness of 360m, comprising mainly pahoehoe flows. Upper Ratangarh formation occupies a large part and comprises a sequence of 7 floes showing sparsely to moderately porphyritic character. The area constitutes a sequence of basaltic lava flows while alluvium occupies a small portion. There are two distinct hydrogeological units' i.e. fissured formations and porous formations (Aher, 2017). Utilization of groundwater is through dug wells and bored wells. The occurrence and movement of groundwater is controlled by variation in water bearing properties of these formations (Fig.2).
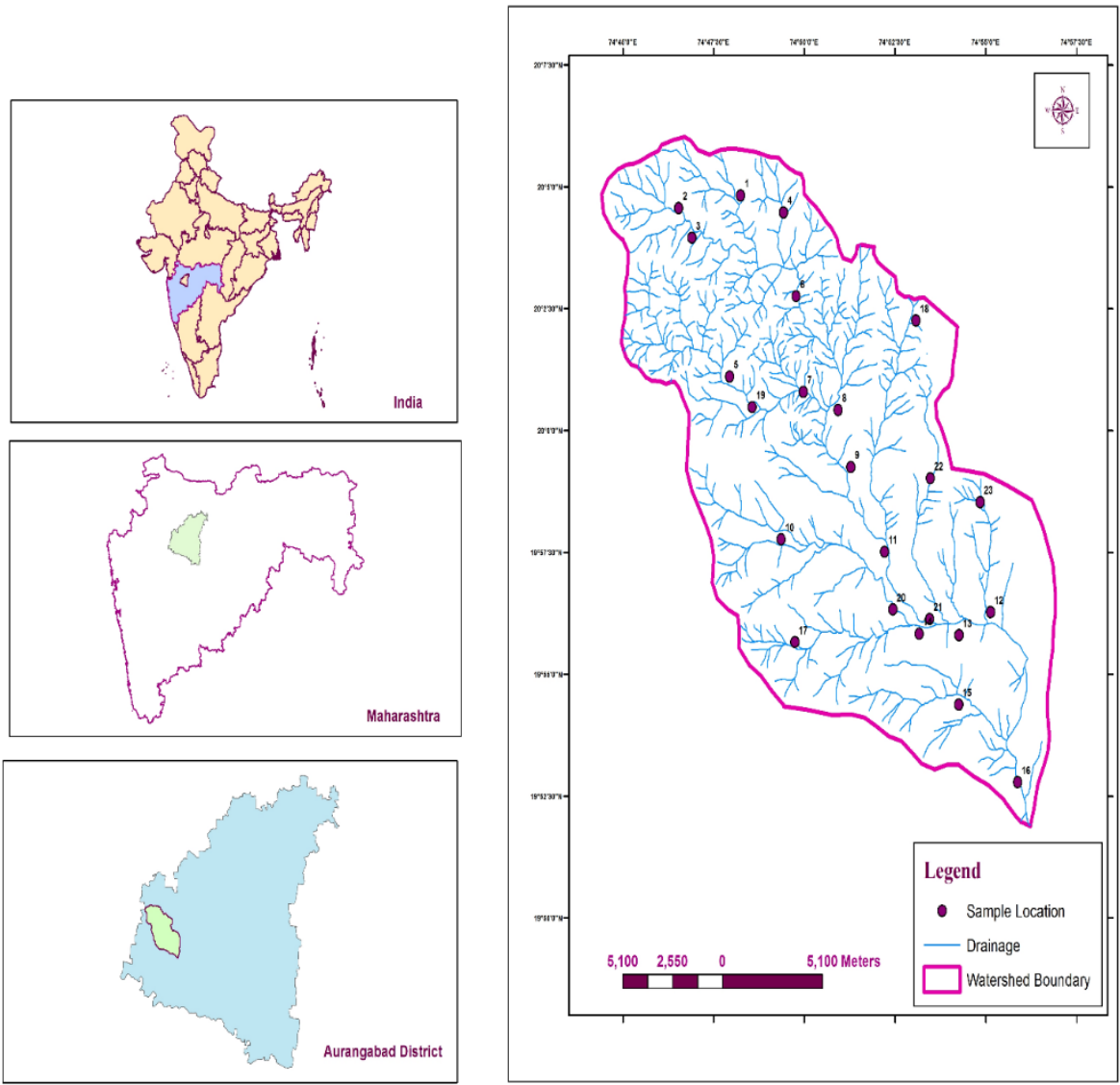

Fig. 1: Location Map of the Study Area. 


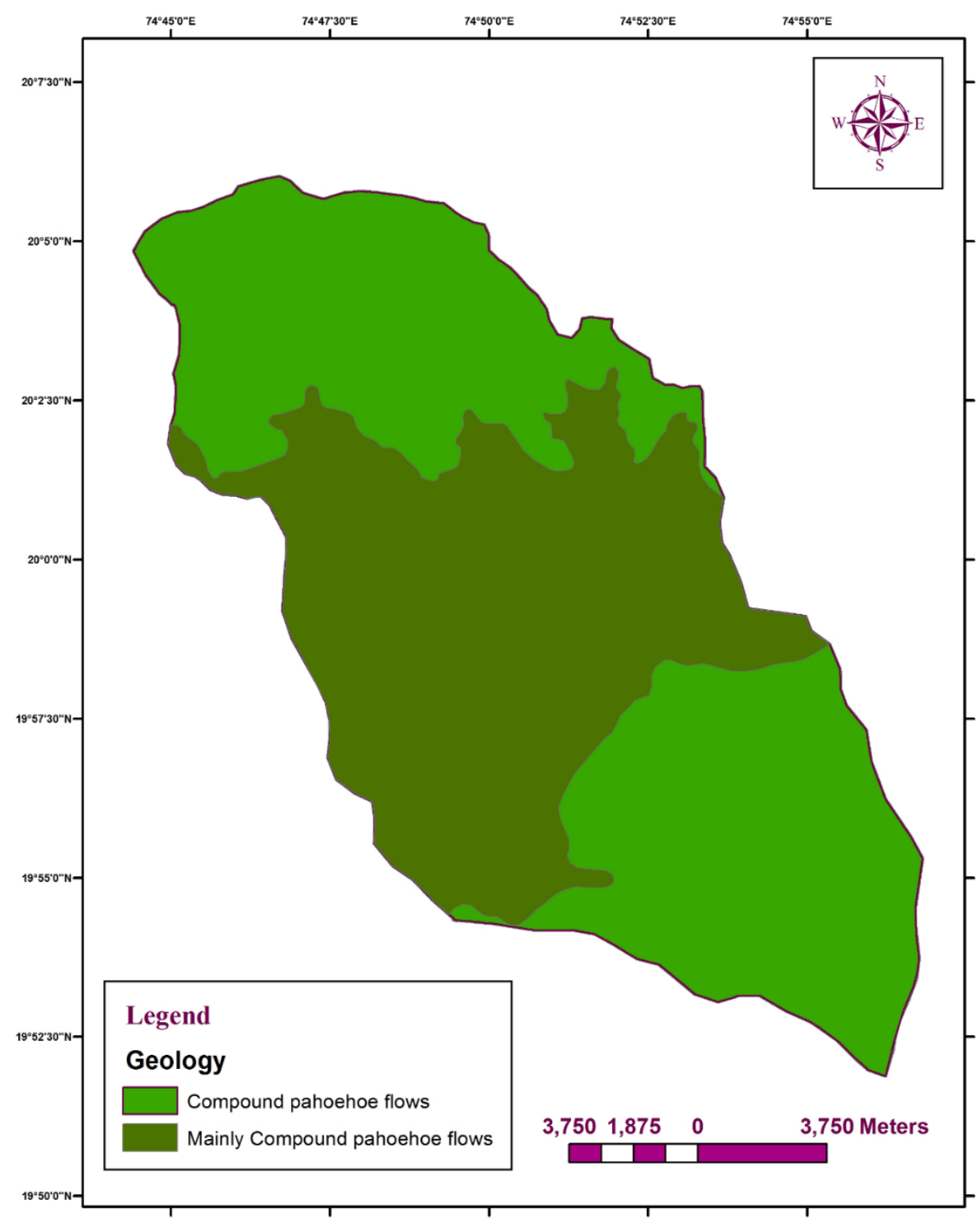

Fig. 2: Geological Map of the Study Area.

\section{Methodology}

The base map of the study area was scanned and digitized from the Survey of India (SOI) Toposheets No. 46L/16 and 46I/13 (1:50,000). ArcGIS: 10 is used to map and analyze the data for the evaluation of groundwater quality. Samples were collected from wells as shown in (Fig. 1.)

The groundwater samples were analysed for major ion chemistry, using of APHA (2017) standard water quality methodology. The physicochemical parameters viz. pH, electrical conductivity (EC), total dissolved solids (TDS), turbidity, total hardness, cations like calcium $(\mathrm{Ca})$, magnesium $(\mathrm{Mg})$, sodium $(\mathrm{Na})$ and potassium $(\mathrm{K})$, and anions like Carbonate $\left(\mathrm{CO}_{3}\right)$, bicarbonate $\left(\mathrm{HCO}_{3}\right)$, total alkalinity, chloride $(\mathrm{Cl})$, sulphate $\left(\mathrm{SO}_{4}\right)$, nitrate $\left(\mathrm{NO}_{3}\right)$ and fluoride $(\mathrm{F})$. The $\mathrm{pH}$ and electrical conductivity $(\mathrm{EC})$ of the groundwater samples were measured in the field. The TDS was calculated from EC adhering to the procedure of Hem (1991). The rest of the parameters were determined in the laboratory immediately after the groundwater sampling. For analytical precision of each groundwater sample, the concentrations of cations and anions were used to compute the ionic-balance-error, which was observed to be within the standard limit of $\pm 10 \%$ (Domenico and Schwartz 1990). The suitability of groundwater for domestic purposes were evaluated by comparing the values of different water quality parameters with those of the World Health Organisation (WHO,2011) and Bureau of Indian standard (BIS,2012) guidelines values for drinking water.

\subsection{Water quality index system}

A water quality index, common with many other indices systems, relates a group of water quality parameters to a common scale and combines them into a single number in accordance with a chosen method of computation. The desired use of WQI is to assess water quality trends for management purpose even though it is not meant for an absolute measure of the degree of pollution or the actual water quality. WQI is defined as an index reflecting the composite influence of different water quality parameters which is considered and taken for calculation of water quality index. The standards for drinking purposes as recommended by BIS, (2012), WHO, (2011), and ICMR, (1975) have been used for the calculation of WQI, which involves three steps In the first step each of the fourteen parameters like $\mathrm{pH}, \mathrm{EC}, \mathrm{TDS}, \mathrm{TH}, \mathrm{Ca}, \mathrm{Mg}, \mathrm{SO} 4, \mathrm{Cl}, \mathrm{NO}$, Na, K, TA. F and Fe were assigned weights $\left(\mathrm{w}_{\mathrm{i}}\right)$ ranging from 2 to 5 , and its selection depends on their significance in quality of water for drinking purposes (Ramakrishnalah et al., 2009). In the second step is relative weights $\left(\mathrm{W}_{\mathrm{i}}\right)$ are calculated through equation (1). 
$\mathrm{W}_{\mathrm{i}}=\frac{\mathrm{w}_{\mathrm{i}}}{\sum_{\mathrm{i}=1}^{\mathrm{n}} \mathrm{W}_{\mathrm{i}}}$

Where $\left(\mathrm{W}_{\mathrm{i}}\right)$ is the relative weight, $\left(\mathrm{W}_{\mathrm{i}}\right)$ is the weight of each parameter and $(\mathrm{n})$ is the number of parameters Table 1 . In the third step, quality rating scale calculation (Qi) for each individual parameter is computed by dividing its concentration for each ground water sample with drinking water quality standards of and then multiplied by 100 using equation (2).

$\mathrm{Q}_{\mathrm{i}}=\left(\mathrm{C}_{\mathrm{i}} / \mathrm{S}_{\mathrm{i}}\right) \times 100$

here $Q_{i}$ is the quality rating, $C_{i}$ is the concentration of each chemical parameter in each water sample in milligrams per litre (mg/L) and $S_{i}$ is the drinking water standard guidelines for each chemical parameter. Eventuality, water quality sub-index (SIi) for each chemical parameter was computed by equation (3), and whole the WQI was determined by equation (4).

$\mathrm{SI}_{\mathrm{i}}=\mathrm{W}_{\mathrm{i}} \times \mathrm{Q}_{\mathrm{i}}$

$\mathrm{WQI}=\sum S \mathrm{I}_{\mathrm{i}-\mathrm{n}}$

Where,

$\mathrm{SI}_{\mathrm{i}}$ is the sub-index of the ith parameter,

$\mathrm{Q}_{\mathrm{i}}$ is the rating based on the concentration of ith parameter, and $\mathrm{n}$ is the total numbers of parameter?

\section{Results and discussion}

The statistics results of the physicochemical analysis in pre- and post-monsoon seasons are presented in table 1 . The assigned weight and relative weight of physicochemical parameters for calculation of WQI are presented in Table 2, quality rating in Table 3 and 4, sub index values in Table 5 and 6, WQI values in Table 7, and Computation of water quality index for groundwater samples based on WQI in Table 8.

Table 1: Descriptive Statistics of Pre-Monsoon and Post-Monsoon Season of the Study Area

\begin{tabular}{|c|c|c|c|c|c|c|c|c|}
\hline Parameter & $\begin{array}{l}\text { pre-monsoon } \\
\text { Minimum }\end{array}$ & Maximum & Mean & Std. Deviation & $\begin{array}{l}\text { post-monsoon } \\
\text { Minimum }\end{array}$ & Maximum & Mean & Std. Deviation \\
\hline $\mathrm{pH}$ & 7.01 & 9.27 & 7.72 & 0.65 & 6.50 & 8.76 & 7.21 & 0.65 \\
\hline EC & 360 & 4360 & 1088 & 828 & 418 & 4418 & 1146 & 828 \\
\hline TDS & 234 & 2834 & 708 & 538 & 272 & 2872 & 745 & 538 \\
\hline Turbidity & 0.10 & 0.90 & 0.69 & 0.18 & 0.01 & 0.79 & 0.58 & 0.18 \\
\hline $\mathrm{TH}$ & 112 & 1164 & 346 & 209 & 147 & 1199 & 381 & 209 \\
\hline $\mathrm{Ca}$ & 14 & 115 & 70 & 29 & 32 & 133 & 88 & 29 \\
\hline $\mathrm{Mg}$ & 6 & 217 & 41 & 42 & 19 & 230 & 54 & 42 \\
\hline $\mathrm{Na}$ & 14 & 122 & 62 & 31 & 29 & 137 & 77 & 31 \\
\hline $\mathrm{K}$ & 0.10 & 138.00 & 6.81 & 28.63 & 0.23 & 138.13 & 6.94 & 28.63 \\
\hline TA & 56 & 756 & 230 & 136 & 92 & 792 & 266 & 136 \\
\hline $\mathrm{CO}_{3}$ & 0.00 & 72.00 & 11.48 & 21.28 & 0.00 & 72.00 & 13.83 & 24.70 \\
\hline $\mathrm{HCO}_{3}$ & 12 & 708 & 218 & 132 & 35 & 731 & 241 & 132 \\
\hline $\mathrm{Cl}$ & 26 & 234 & 129 & 59 & 52 & 260 & 155 & 59 \\
\hline $\mathrm{SO}_{4}$ & 19 & 289 & 60 & 56 & 48 & 318 & 89 & 56 \\
\hline $\mathrm{F}$ & 0.07 & 1.92 & 0.74 & 0.58 & 0.08 & 1.77 & 0.59 & 0.58 \\
\hline $\mathrm{NO}_{3}$ & 11 & 155 & 44 & 31 & 22 & 166 & 55 & 31 \\
\hline $\mathrm{Fe}$ & 0.01 & 1.35 & 0.54 & 0.54 & 0.02 & 1.36 & 0.55 & 0.54 \\
\hline WQI & 45.37 & 333.55 & 104.91 & 61.28 & 57.11 & 345.29 & 116.65 & 61.28 \\
\hline
\end{tabular}

Table 2: The Assigned Weight and Relative Weight of Physicochemical Parameters

\begin{tabular}{|c|c|c|c|c|c|c|}
\hline Sr. No. & Parameter & Units & Drinking water Standards (Si) & Recommending Agency & weight (wi) & Relative weight (Wi) \\
\hline 1 & $\mathrm{pH}$ & - & $6.5-8.5$ & ICMR/BIS & 3 & 0.0625 \\
\hline 2 & $\mathrm{EC}$ & $\mu / \mathrm{cm}$ & 300 & ICMR & 3 & 0.0625 \\
\hline 3 & TDS & $\mathrm{mg} / \mathrm{L}$ & 500 & ICMR/BIS & 5 & 0.1042 \\
\hline 4 & $\mathrm{TH}$ & $\mathrm{mg} / \mathrm{L}$ & 300 & BIS & 3 & 0.0625 \\
\hline 5 & $\mathrm{Ca}$ & $\mathrm{mg} / \mathrm{L}$ & 75 & ICMR/BIS & 3 & 0.0625 \\
\hline 6 & $\mathrm{Mg}$ & $\mathrm{mg} / \mathrm{L}$ & 30 & ICMR/BIS & 3 & 0.0625 \\
\hline 7 & $\mathrm{SO}_{4}$ & $\mathrm{mg} / \mathrm{L}$ & 200 & BIS & 3 & 0.0625 \\
\hline 8 & $\mathrm{Cl}$ & $\mathrm{mg} / \mathrm{L}$ & 250 & ICMR/BIS & 4 & 0.0833 \\
\hline 9 & $\mathrm{NO}_{3}$ & $\mathrm{mg} / \mathrm{L}$ & 45 & ICMR/BIS & 5 & 0.1042 \\
\hline 10 & $\mathrm{Na}$ & $\mathrm{mg} / \mathrm{L}$ & 200 & WHO & 2 & 0.0417 \\
\hline 11 & $\mathrm{~K}$ & $\mathrm{mg} / \mathrm{L}$ & 12 & WHO & 2 & 0.0417 \\
\hline 12 & TA & $\mathrm{mg} / \mathrm{L}$ & 200 & BIS & 3 & 0.0625 \\
\hline 13 & $\mathrm{~F}$ & $\mathrm{mg} / \mathrm{L}$ & 1.5 & BIS/WHO & 5 & 0.1042 \\
\hline 14 & $\mathrm{Fe}$ & $\mathrm{mg} / \mathrm{L}$ & 1.0 & BIS/WHO & 4 & 0.0833 \\
\hline & & & & & $\sum 48$ & $\sum 1.00$ \\
\hline
\end{tabular}

\section{1. $\mathrm{pH}$}

$\mathrm{pH}$ is one of the important factors of ground water. $\mathrm{pH}$ is ranging from 7.01 to 9.27 and 6.50 to 8.76 during pre- and post-monsoon seasons, respectively. The slightly increase of $\mathrm{pH}$ in pre -monsoon season can be attributed to the discontinued supply of $\mathrm{CO}_{2}$ (Pondhe et al., 1997). $9 \%$ and $4 \%$ samples were exceeding the permissible limit in pre- and post-monsoon season, respectively prescribed by BIS (2012). The study indicating that groundwater is slightly alkaline in nature. 
Table 3: Water Quality Rating (Qi) Values of Physicochemical Parameters (Pre-Monsoon Season)

\begin{tabular}{|c|c|c|c|c|c|c|c|c|c|c|c|c|c|c|}
\hline $\begin{array}{l}\text { Samples } \\
\text { No }\end{array}$ & $\mathrm{pH}$ & $\mathrm{EC}$ & TDS & $\mathrm{TH}$ & $\mathrm{Ca}$ & $\mathrm{Mg}$ & TA & $\mathrm{Cl}$ & $\mathrm{SO}_{4}$ & $\mathrm{~F}$ & $\mathrm{NO}_{3}$ & $\mathrm{Na}$ & $\mathrm{K}$ & $\mathrm{Fe}$ \\
\hline 1 & 85.88 & 266.67 & 104 & 134 & 123.73 & 29.16 & 106 & 56.8 & 21.96 & 72 & 50.11 & 4.37 & 3.33 & 9 \\
\hline 2 & 82.59 & 280 & 109.2 & 156 & 87.47 & 119.88 & 100 & 60 & 38 & 34.67 & 80.82 & 17.98 & 0.83 & 6 \\
\hline 3 & 84.71 & 273.33 & 106.6 & 142 & 68.27 & 126.36 & 124 & 43.2 & 13.5 & 52 & 54.83 & 18.95 & 7.5 & 7 \\
\hline 4 & 84.35 & 160 & 62.4 & 98 & 29.87 & 113.4 & 70 & 10.4 & 21 & 73.33 & 50.99 & 17.01 & 3.33 & 11 \\
\hline 5 & 96.94 & 389.33 & 151.84 & 226 & 117.33 & 187.92 & 100 & 65.6 & 32.9 & 16.67 & 131.52 & 28.19 & 8.33 & 122 \\
\hline 6 & 98.24 & 636 & 248.04 & 264 & 136.53 & 220.32 & 128 & 83.2 & 59.79 & 23.33 & 170.01 & 33.05 & 13.33 & 132 \\
\hline 7 & 96.47 & 291.67 & 113.75 & 180 & 102.4 & 136.08 & 98 & 53.6 & 24.83 & 27.33 & 54.14 & 20.41 & 7.5 & 49 \\
\hline 8 & 90 & 503.33 & 196.3 & 244 & 119.47 & 213.84 & 128 & 79.2 & 28.56 & 20.67 & 127.98 & 32.08 & 4.17 & 107 \\
\hline 9 & 109.06 & 120 & 46.8 & 80 & 25.6 & 90.72 & 30 & 23.2 & 14.5 & 26 & 90.57 & 13.61 & 48.33 & 51 \\
\hline 10 & 105.65 & 293.33 & 114.4 & 176 & 83.2 & 158.76 & 104 & 57.6 & 20.67 & 4.67 & 109.77 & 23.81 & 0.83 & 94 \\
\hline 11 & 82.71 & 296.67 & 115.7 & 148 & 110.93 & 71.28 & 98 & 46.4 & 22.2 & 6 & 80.33 & 10.69 & 2.5 & 101 \\
\hline 12 & 85.76 & 576.67 & 224.9 & 196 & 91.73 & 178.2 & 194 & 89.6 & 35.53 & 6.67 & 154.26 & 26.73 & 1150 & 120 \\
\hline 13 & 87.29 & 473.33 & 184.6 & 196 & 153.6 & 84.24 & 128 & 93.6 & 32.62 & 12 & 138.51 & 12.64 & 3.33 & 122 \\
\hline 14 & 82.47 & 266.67 & 104 & 124 & 119.47 & 19.44 & 86 & 31.2 & 13.83 & 14 & 113.01 & 2.92 & 2.5 & 125 \\
\hline 15 & 85.41 & 280 & 109.2 & 136 & 66.13 & 119.88 & 104 & 46.4 & 16.7 & 22.67 & 61.92 & 17.98 & 2.5 & 135 \\
\hline 16 & 88.94 & 516 & 201.24 & 242 & 140.8 & 178.2 & 170 & 64.8 & 50.02 & 64.67 & 154.95 & 26.73 & 28.33 & 1 \\
\hline 17 & 92.35 & 259.33 & 101.14 & 146 & 100.27 & 84.24 & 120 & 49.6 & 16.76 & 80 & 61.43 & 12.64 & 1.67 & 5 \\
\hline 18 & 83.65 & 159.33 & 62.14 & 98 & 51.2 & 81 & 84 & 27.2 & 9.61 & 128 & 42.04 & 12.15 & 2.5 & 4 \\
\hline 19 & 86.12 & 120 & 46.8 & 56 & 19.2 & 61.56 & 28 & 22.4 & 16.33 & 106 & 25.2 & 9.23 & 0.83 & 4 \\
\hline 20 & 89.65 & 216.67 & 84.5 & 116 & 72.53 & 77.76 & 100 & 17.6 & 12 & 120 & 82.69 & 11.66 & 4.17 & 8 \\
\hline 21 & 93.06 & 229.33 & 89.44 & 116 & 78.93 & 68.04 & 76 & 48.8 & 22.75 & 103.33 & 68.81 & 10.21 & 6.67 & 8 \\
\hline 22 & 99.41 & 1453.33 & 566.8 & 582 & 145.07 & 722.52 & 378 & 80.8 & 144.5 & 58 & 344.65 & 108 & 1.67 & 11 \\
\hline 23 & 98.59 & 283.33 & 110.5 & 124 & 113.07 & 29.16 & 90 & 36 & 20.98 & 61.33 & 24.22 & 4.37 & 0.83 & 12 \\
\hline
\end{tabular}

Table 4: Water Quality Rating (Qi) Values of Physicochemical Parameters (Post-Monsoon Season)

\begin{tabular}{|c|c|c|c|c|c|c|c|c|c|c|c|c|c|c|}
\hline Samples No & $\mathrm{pH}$ & $\mathrm{EC}$ & TDS & $\mathrm{TH}$ & $\mathrm{Ca}$ & $\mathrm{Mg}$ & TA & $\mathrm{Cl}$ & $\mathrm{SO}_{4}$ & $\mathrm{~F}$ & $\mathrm{NO}_{3}$ & $\mathrm{Na}$ & $\mathrm{K}$ & $\mathrm{Fe}$ \\
\hline 1 & 79.88 & 286 & 111.54 & 151.5 & 147.73 & 72.49 & 124 & 67.2 & 36.46 & 62 & 74.55 & 10.87 & 4.42 & 10 \\
\hline 2 & 76.59 & 299.33 & 116.74 & 173.5 & 111.47 & 163.21 & 118 & 70.4 & 52.5 & 24.67 & 105.27 & 24.48 & 1.92 & 7 \\
\hline 3 & 78.71 & 292.67 & 114.14 & 159.5 & 92.27 & 169.69 & 142 & 53.6 & 28 & 42 & 79.28 & 25.45 & 8.58 & 8 \\
\hline 4 & 78.35 & 179.33 & 69.94 & 115.5 & 53.87 & 156.73 & 88 & 20.8 & 35.5 & 63.33 & 75.44 & 23.51 & 4.42 & 12 \\
\hline 5 & 90.94 & 408.67 & 159.38 & 243.5 & 141.33 & 231.25 & 118 & 76 & 47.4 & 6.67 & 155.97 & 34.69 & 9.42 & 123 \\
\hline 6 & 92.24 & 655.33 & 255.58 & 281.5 & 160.53 & 263.65 & 146 & 93.6 & 74.29 & 13.33 & 194.46 & 39.55 & 14.4 & 133 \\
\hline 7 & 90.47 & 311 & 121.29 & 197.5 & 126.4 & 179.41 & 116 & 64 & 39.33 & 17.33 & 78.59 & 26.91 & 8.58 & 50 \\
\hline 8 & 84 & 522.67 & 203.84 & 261.5 & 143.47 & 257.17 & 146 & 89.6 & 43.06 & 10.67 & 152.42 & 38.58 & 5.25 & 108 \\
\hline 9 & 103.06 & 139.33 & 54.34 & 97.5 & 49.6 & 134.05 & 48 & 33.6 & 29 & 16 & 115.01 & 20.11 & 49.4 & 52 \\
\hline 10 & 99.65 & 312.67 & 121.94 & 193.5 & 107.2 & 202.09 & 122 & 68 & 35.17 & 5.33 & 134.21 & 30.31 & 1.92 & 95 \\
\hline 11 & 76.71 & 316 & 123.24 & 165.5 & 134.93 & 114.61 & 116 & 56.8 & 36.7 & 4 & 104.78 & 17.19 & 3.58 & 102 \\
\hline 12 & 79.76 & 596 & 232.44 & 213.5 & 115.73 & 221.53 & 212 & 100 & 50.03 & 3.33 & 178.71 & 33.23 & 1151 & 121 \\
\hline 13 & 81.29 & 492.67 & 192.14 & 213.5 & 177.6 & 127.57 & 146 & 104 & 47.12 & 2 & 162.96 & 19.14 & 4.42 & 123 \\
\hline 14 & 76.47 & 286 & 111.54 & 141.5 & 143.47 & 62.77 & 104 & 41.6 & 28.33 & 4 & 137.46 & 9.42 & 3.58 & 126 \\
\hline 15 & 79.41 & 299.33 & 116.74 & 153.5 & 90.13 & 163.21 & 122 & 56.8 & 31.2 & 12.67 & 86.37 & 24.48 & 3.58 & 136 \\
\hline 16 & 82.94 & 535.33 & 208.78 & 259.5 & 164.8 & 221.53 & 188 & 75.2 & 64.52 & 54.67 & 179.4 & 33.23 & 29.4 & 2 \\
\hline 17 & 86.35 & 278.67 & 108.68 & 163.5 & 124.27 & 127.57 & 138 & 60 & 31.26 & 70 & 85.87 & 19.14 & 2.75 & 6 \\
\hline 18 & 77.65 & 178.67 & 69.68 & 115.5 & 75.2 & 124.33 & 102 & 37.6 & 24.11 & 118 & 66.48 & 18.65 & 3.58 & 5 \\
\hline 19 & 80.12 & 139.33 & 54.34 & 73.5 & 43.2 & 104.89 & 46 & 32.8 & 30.83 & 96 & 49.65 & 15.73 & 1.92 & 5 \\
\hline 20 & 83.65 & 236 & 92.04 & 133.5 & 96.53 & 121.09 & 118 & 28 & 26.5 & 110 & 107.14 & 18.16 & 5.25 & 9 \\
\hline 21 & 87.06 & 248.67 & 96.98 & 133.5 & 102.93 & 111.37 & 94 & 59.2 & 37.25 & 93.33 & 93.26 & 16.71 & 7.75 & 9 \\
\hline 22 & 93.41 & 1472.6 & 574.34 & 599.5 & 169.07 & 765.85 & 396 & 91.2 & 159 & 48 & 369.1 & 114.9 & 2.75 & 12 \\
\hline 23 & 92.59 & 302.67 & 118.04 & 141.5 & 137.07 & 72.49 & 108 & 46.4 & 35.48 & 51.33 & 48.66 & 10.87 & 1.92 & 13 \\
\hline
\end{tabular}

Table 5: Calculated Sub Index (Sli) Values of Physicochemical Parameters (Pre-Monsoon Season)

\begin{tabular}{|c|c|c|c|c|c|c|c|c|c|c|c|c|c|c|}
\hline Samples No & $\mathrm{pH}$ & $\mathrm{EC}$ & TDS & $\mathrm{TH}$ & $\mathrm{Ca}$ & $\mathrm{Mg}$ & TA & $\mathrm{Cl}$ & $\mathrm{SO}_{4}$ & $\mathrm{~F}$ & $\mathrm{NO}_{3}$ & $\mathrm{Na}$ & $\mathrm{K}$ & $\mathrm{Fe}$ \\
\hline 1 & 5.4 & 16.7 & 10.8 & 8.4 & 7.7 & 1.8 & 6.6 & 4.7 & 1.4 & 7.5 & 5.2 & 0.2 & 0.1 & 0.7 \\
\hline 2 & 5.2 & 17.5 & 11.4 & 9.8 & 5.5 & 7.5 & 6.3 & 5 & 2.4 & 3.6 & 8.4 & 0.7 & 0 & 0.5 \\
\hline 3 & 5.3 & 17.1 & 11.1 & 8.9 & 4.3 & 7.9 & 7.8 & 3.6 & 0.8 & 5.4 & 5.7 & 0.8 & 0.3 & 0.6 \\
\hline 4 & 5.3 & 10 & 6.5 & 6.1 & 1.9 & 7.1 & 4.4 & 0.9 & 1.3 & 7.6 & 5.3 & 0.7 & 0.1 & 0.9 \\
\hline 5 & 6.1 & 24.3 & 15.8 & 14.1 & 7.3 & 11.7 & 6.3 & 5.5 & 2.1 & 1.7 & 13.7 & 1.2 & 0.3 & 10.2 \\
\hline 6 & 6.1 & 39.8 & 25.8 & 16.5 & 8.5 & 13.8 & 8 & 6.9 & 3.7 & 2.4 & 17.7 & 1.4 & 0.6 & 11 \\
\hline 7 & 6 & 18.2 & 11.9 & 11.3 & 6.4 & 8.5 & 6.1 & 4.5 & 1.6 & 2.8 & 5.6 & 0.9 & 0.3 & 4.1 \\
\hline 8 & 5.6 & 31.5 & 20.5 & 15.3 & 7.5 & 13.4 & 8 & 6.6 & 1.8 & 2.2 & 13.3 & 1.3 & 0.2 & 8.9 \\
\hline 9 & 6.8 & 7.5 & 4.9 & 5 & 1.6 & 5.7 & 1.9 & 1.9 & 0.9 & 2.7 & 9.4 & 0.6 & 2 & 4.2 \\
\hline 10 & 6.6 & 18.3 & 11.9 & 11 & 5.2 & 9.9 & 6.5 & 4.8 & 1.3 & 0.5 & 11.4 & 1 & 0 & 7.8 \\
\hline 11 & 5.2 & 18.5 & 12.1 & 9.3 & 6.9 & 4.5 & 6.1 & 3.9 & 1.4 & 0.6 & 8.4 & 0.4 & 0.1 & 8.4 \\
\hline 12 & 5.4 & 36 & 23.4 & 12.3 & 5.7 & 11.1 & 12.1 & 7.5 & 2.2 & 0.7 & 16.1 & 1.1 & 48 & 10 \\
\hline 13 & 5.5 & 29.6 & 19.2 & 12.3 & 9.6 & 5.3 & 8 & 7.8 & 2 & 1.3 & 14.4 & 0.5 & 0.1 & 10.2 \\
\hline 14 & 5.2 & 16.7 & 10.8 & 7.8 & 7.5 & 1.2 & 5.4 & 2.6 & 0.9 & 1.5 & 11.8 & 0.1 & 0.1 & 10.4 \\
\hline 15 & 5.3 & 17.5 & 11.4 & 8.5 & 4.1 & 7.5 & 6.5 & 3.9 & 1 & 2.4 & 6.5 & 0.7 & 0.1 & 11.2 \\
\hline 16 & 5.6 & 32.3 & 21 & 15.1 & 8.8 & 11.1 & 10.6 & 5.4 & 3.1 & 6.7 & 16.1 & 1.1 & 1.2 & 0.1 \\
\hline 17 & 5.8 & 16.2 & 10.5 & 9.1 & 6.3 & 5.3 & 7.5 & 4.1 & 1 & 8.3 & 6.4 & 0.5 & 0.1 & 0.4 \\
\hline 18 & 5.2 & 10 & 6.5 & 6.1 & 3.2 & 5.1 & 5.3 & 2.3 & 0.6 & 13.3 & 4.4 & 0.5 & 0.1 & 0.3 \\
\hline 19 & 5.4 & 7.5 & 4.9 & 3.5 & 1.2 & 3.8 & 1.8 & 1.9 & 1 & 11 & 2.6 & 0.4 & 0 & 0.3 \\
\hline 20 & 5.6 & 13.5 & 8.8 & 7.3 & 4.5 & 4.9 & 6.3 & 1.5 & 0.7 & 12.5 & 8.6 & 0.5 & 0.2 & 0.7 \\
\hline 21 & 5.8 & 14.3 & 9.3 & 7.3 & 4.9 & 4.3 & 4.8 & 4.1 & 1.4 & 10.8 & 7.2 & 0.4 & 0.3 & 0.7 \\
\hline 22 & 6.2 & 90.8 & 59.1 & 36.4 & 9.1 & 45.2 & 23.6 & 6.7 & 9 & 6 & 35.9 & 4.5 & 0.1 & 0.9 \\
\hline 23 & 6.2 & 17.7 & 11.5 & 7.8 & 7.1 & 1.8 & 5.6 & 3 & 1.3 & 6.4 & 2.5 & 0.2 & 0 & 1 \\
\hline
\end{tabular}


Table 6: Calculated Sub Index (Sli) Values of Physicochemical Parameters (Post-Monsoon Season)

\begin{tabular}{|c|c|c|c|c|c|c|c|c|c|c|c|c|c|c|}
\hline $\begin{array}{l}\text { Samples } \\
\text { No }\end{array}$ & $\mathrm{pH}$ & $\mathrm{EC}$ & TDS & $\mathrm{TH}$ & $\mathrm{Ca}$ & $\mathrm{Mg}$ & TA & $\mathrm{Cl}$ & $\mathrm{SO}_{4}$ & $\mathrm{~F}$ & $\mathrm{NO}_{3}$ & $\mathrm{Na}$ & $\mathrm{K}$ & $\mathrm{Fe}$ \\
\hline 1 & 5 & 17.9 & 11.6 & 9.5 & 9.2 & 4.5 & 7.8 & 5.6 & 2.3 & 6.5 & 7.8 & 0.5 & 0.2 & 0.8 \\
\hline 2 & 4.8 & 18.7 & 12.2 & 10.8 & 7 & 10.2 & 7.4 & 5.9 & 3.3 & 2.6 & 11 & 1 & 0.1 & 0.6 \\
\hline 3 & 4.9 & 18.3 & 11.9 & 10 & 5.8 & 10.6 & 8.9 & 4.5 & 1.8 & 4.4 & 8.3 & 1.1 & 0.4 & 0.7 \\
\hline 4 & 4.9 & 11.2 & 7.3 & 7.2 & 3.4 & 9.8 & 5.5 & 1.7 & 2.2 & 6.6 & 7.9 & 1 & 0.2 & 1 \\
\hline 5 & 5.7 & 25.5 & 16.6 & 15.2 & 8.8 & 14.5 & 7.4 & 6.3 & 3 & 0.7 & 16.3 & 1.4 & 0.4 & 10.3 \\
\hline 6 & 5.8 & 41 & 26.6 & 17.6 & 10 & 16.5 & 9.1 & 7.8 & 4.6 & 1.4 & 20.3 & 1.6 & 0.6 & 11.1 \\
\hline 7 & 5.7 & 19.4 & 12.6 & 12.3 & 7.9 & 11.2 & 7.3 & 5.3 & 2.5 & 1.8 & 8.2 & 1.1 & 0.4 & 4.2 \\
\hline 8 & 5.3 & 32.7 & 21.2 & 16.3 & 9 & 16.1 & 9.1 & 7.5 & 2.7 & 1.1 & 15.9 & 1.6 & 0.2 & 9 \\
\hline 9 & 6.4 & 8.7 & 5.7 & 6.1 & 3.1 & 8.4 & 3 & 2.8 & 1.8 & 1.7 & 12 & 0.8 & 2.1 & 4.3 \\
\hline 10 & 6.2 & 19.5 & 12.7 & 12.1 & 6.7 & 12.6 & 7.6 & 5.7 & 2.2 & 0.6 & 14 & 1.3 & 0.1 & 7.9 \\
\hline 11 & 4.8 & 19.8 & 12.8 & 10.3 & 8.4 & 7.2 & 7.3 & 4.7 & 2.3 & 0.4 & 10.9 & 0.7 & 0.1 & 8.5 \\
\hline 12 & 5 & 37.3 & 24.2 & 13.3 & 7.2 & 13.8 & 13.3 & 8.3 & 3.1 & 0.3 & 18.6 & 1.4 & 48 & 10.1 \\
\hline 13 & 5.1 & 30.8 & 20 & 13.3 & 11.1 & 8 & 9.1 & 8.7 & 2.9 & 0.2 & 17 & 0.8 & 0.2 & 10.3 \\
\hline 14 & 4.8 & 17.9 & 11.6 & 8.8 & 9 & 3.9 & 6.5 & 3.5 & 1.8 & 0.4 & 14.3 & 0.4 & 0.1 & 10.5 \\
\hline 15 & 5 & 18.7 & 12.2 & 9.6 & 5.6 & 10.2 & 7.6 & 4.7 & 2 & 1.3 & 9 & 1 & 0.1 & 11.3 \\
\hline 16 & 5.2 & 33.5 & 21.8 & 16.2 & 10.3 & 13.8 & 11.8 & 6.3 & 4 & 5.7 & 18.7 & 1.4 & 1.2 & 0.2 \\
\hline 17 & 5.4 & 17.4 & 11.3 & 10.2 & 7.8 & 8 & 8.6 & 5 & 2 & 7.3 & 8.9 & 0.8 & 0.1 & 0.5 \\
\hline 18 & 4.9 & 11.2 & 7.3 & 7.2 & 4.7 & 7.8 & 6.4 & 3.1 & 1.5 & 12.3 & 6.9 & 0.8 & 0.1 & 0.4 \\
\hline 19 & 5 & 8.7 & 5.7 & 4.6 & 2.7 & 6.6 & 2.9 & 2.7 & 1.9 & 10 & 5.2 & 0.7 & 0.1 & 0.4 \\
\hline 20 & 5.2 & 14.8 & 9.6 & 8.3 & 6 & 7.6 & 7.4 & 2.3 & 1.7 & 11.5 & 11.2 & 0.8 & 0.2 & 0.8 \\
\hline 21 & 5.4 & 15.5 & 10.1 & 8.3 & 6.4 & 7 & 5.9 & 4.9 & 2.3 & 9.7 & 9.7 & 0.7 & 0.3 & 0.8 \\
\hline 22 & 5.8 & 92 & 59.8 & 37.5 & 10.6 & 47.9 & 24.8 & 7.6 & 9.9 & 5 & 38.5 & 4.8 & 0.1 & 1 \\
\hline 23 & 5.8 & 18.9 & 12.3 & 8.8 & 8.6 & 4.5 & 6.8 & 3.9 & 2.2 & 5.3 & 5.1 & 0.5 & 0.1 & 1.1 \\
\hline
\end{tabular}

\subsection{Electrical conductivity}

The EC values ranges from 360 to $4360 \mu / \mathrm{cm}$ and 418 to $4418 \mu / \mathrm{cm}$ during pre- and post-monsoon respectively. The EC of the ground water sample in the post-monsoon season are higher as compare to the EC of pre-monsoon season which can be related to the dissolution of minerals (Ballukraya and Ravi, 1999). The maximum limit of EC in drinking water is prescribed as $1500 \mu / \mathrm{cm}$ as per WHO standard (2011). $22 \%$ samples were exceeded the permissible limit, during pre- and post-monsoon season

\subsection{Total Dissolves solids}

Total dissolves solid is the concentration of all dissolved minerals in water indicating the general nature of salinity of water. It ranged from 234 to $3834 \mathrm{mg} / \mathrm{L}$ for pre -monsoon and 271 to $2871 \mathrm{mg} / \mathrm{L}$ in post-monsoon. Only $4 \%$ samples were exceeding maximum permissible limit. prescribed by the BIS (2012) in both seasons. The TDS of ground water sample in the post-monsoon season was higher as compared to the pre-monsoon season which may be attributed due to leaching of salts from ground surface by recharge. TDS in ground water originate from natural sources and sewage (Kurian, 2001; Gaikwad et al, 2019). Higher concentration of dissolved solids may produce distress in cattle and livestock and a salty to water (Srivastava, et al, 2007).

\subsection{Total hardness}

Hardness values were recorded between 112 to $1164 \mathrm{mg} / \mathrm{L}$ and 147 to 1199 during pre- and post-monsoon seasons, respectively. In both the seasons only, one samples were exceeding maximum permissible limit prescribed by the BIS (2012). Sawyer and McCarty (1967) classified groundwater, based on TH, as follows groundwaters with TH $<75,75-150,150-300$ and $>300$ mg/L, has been designated as soft, moderately hard, hard and very hard, respectively. According to the above categorization, $4 \%$ belongs to moderately hard in both season; $52 \%$ and $30 \%$ belongs to hard and the remaining $43 \%$ and $65 \%$ comprises very hard water in the area of this study during preand post-monsoon season respectively. The analytical result indicates that the water in the study area is moderately hard to very hard and such hardness is due to the presence of alkaline earths such as calcium and magnesium.

\subsection{Turbidity}

The values of turbidity are within 0.10 to $0.90 \mathrm{NTU}$ and 0.0 to $0.79 \mathrm{NTU}$ in pre- and post-monsoon season respectively. The permissible turbidity prescribed as a standard for drinking water is between 1 to 5 NTU (BIS, 2012), and all the samples are within prescribed limit.

\subsection{Calcium and magnesium}

The determination of calcium is usually required for potable water and Magnesium is an essential element for man. The values of calcium varied in the range of 14 to $115 \mathrm{mg} / \mathrm{L}$ and .32 to $133 \mathrm{mg} / \mathrm{L}$ in pre- and post-monsoon seasons respectively similarly magnesium values varied within range of 6 to $217 \mathrm{mg} / \mathrm{L}$ in pre-monsoon and 19 to $230 \mathrm{mg} / \mathrm{L}$ in post-monsoon seasons. Both calcium and magnesium are within limit in both seasons given by BIS (2012).

\subsection{Sodium and potassium}

Concentrations of sodium were found to vary within 14 to $122 \mathrm{mg} / \mathrm{L}$ and 29 to $137 \mathrm{mg} / \mathrm{L}$ during pre- and post-monsoon season respectively. Sodium values are within the limit given by BIS (2012), and only one sample of potassium are exceeding the maximum permissible limit of BIS (2012) in both pre- and post-monsoon season. 


\subsection{Total alkalinity, carbonate, and bicarbonate}

Alkalinity is the measure of the capacity of the water to neutralize a strong acid. The Alkalinity in the water is generally imparted by the salts of carbonates, silicates, etc. together with the hydroxyl ions in free State. Most of the natural waters contain substantial amounts of dissolved carbon dioxide, which is the principal source of alkalinity (Trivedy and Goel,1984; Deshpande et al,2012)

The total alkalinity varies from 56 to $756 \mathrm{mg} / \mathrm{L}$ and 92 to $792 \mathrm{mg} / \mathrm{L}$ in pre- and post-monsoon season respectively, the carbonate values 0 to $72 \mathrm{mg} / \mathrm{L}$ during both seasons, and the bicarbonate alkalinity content of the groundwater ranged from 12 to $708 \mathrm{mg} / \mathrm{L}$ and 35 to $731 \mathrm{mg} / \mathrm{L}$ during pre- and post-monsoon season respectively.

\subsection{Chloride}

Concentrations of chloride were found to vary within 26 to $234 \mathrm{mg} / \mathrm{L}$ and 52 to $260 \mathrm{mg} / \mathrm{L}$ in pre- and post-monsoon seasons respectively. Though chloride concentration is high in ground water, but it is below permissible limit of BIS (2012).

\subsection{Sulphate}

The values of sulphate ranged within 19 to $289 \mathrm{mg} / \mathrm{L}$ and 48 to 318 in pre- and post-monsoon season respectively. The maximum values observed can be attributed to addition of septic tank water. Sulphate may have laxative effect if magnesium is present at an equivalent concentration (Chatterjee \& Raziuddin 2002). In the present study sulphate in groundwaters is below permissible limit of BIS (2012).

\subsection{Fluoride}

Concentrations of fluoride were found to vary within 0.07 to $1.92 \mathrm{mg} / \mathrm{L}$ and 0.08 to $1.77 \mathrm{mg} / \mathrm{L}$ in pre- and post-monsoon seasons respectively. $17 \%$ and $9 \%$ samples were exceeding the permissible limit in pre- and post-monsoon season, respectively prescribed by BIS (2012). Fluoride is higher during pre-monsoon season $(1.92 \mathrm{mg} / \mathrm{L})$ due to leaching from fluoride rich rocks and easier accessibility of rain water to weathered rock, long-term irrigation processes, semi-arid climate and long residence time of groundwater (Datta et al. 1996; Robinson and Edington 1946; Hem 1991; Srinivasamoorthy et al. 2008; 2012).

\subsection{Nitrate}

Nitrates are the end products of decomposition of organic matter present in fully oxidised waters and harmful above $45 \mathrm{mg} / \mathrm{L}$. The recommended limit (BIS,2012) of $\mathrm{NO}_{3}$ in drinking water is $45 \mathrm{mg} / \mathrm{L}$ and the concentration is higher in post-monsoon season $(166 \mathrm{mg} / \mathrm{L}) .39 \%$ and 57\% samples were exceeding the permissible limit in pre- and post-monsoon season, respectively prescribed by BIS (2012). High concentration nitrate can cause for methemoglobinemia (an often-fatal disease in infants; the so-called blue baby syndrome). Thus, limited physiological problem (methemoglobinemia) may be associated with the use of groundwater in the study area. The origin of $\mathrm{NO}_{3}$ is derived from agricultural areas due to leaching process from plant nutrient and nitrate fertilizers (Freeze and Cherry 1979; Madison and Brunett 1984).

\subsection{Iron}

The concentration of iron in ground water varies 0.01 to $1.35 \mathrm{mg} / \mathrm{L}$ in pre-monsoon and 0.02 to $1.36 \mathrm{mg} / \mathrm{L}$ in post-monsoon season. The Bureau of Indian Standards has recommended $0.1 \mathrm{mg} / \mathrm{L}$ as the limit for drinking water (BIS 2012). About $35 \%$ and $4 \%$ of the sample of the study area exceeds the maximum permissible limit of $1.0 \mathrm{mg} / \mathrm{L}$ during pre- and post-monsoon season respectively. High concentrations of iron generally cause inky flavour, bitter, and astringent taste. The high value may be due to rusting of casing pipes, non-usage of bore wells for long periods and disposal of scrap iron in open areas due to industrial and allied activity (Deshpande et al, 2013).

Table 7: Status of Water Quality Based on Water Quality Index (WQI)

\begin{tabular}{|c|c|c|c|c|}
\hline Sr. No. & WQI & Category & pre-monsoon $\%$ & post-monsoon $\%$ \\
\hline 1 & $<50$ & Excellent & 4 & 0 \\
\hline 2 & $50-100$ & Good & 65 & 65 \\
\hline 3 & $100-200$ & Poor & 26 & 26 \\
\hline 4 & $200-300$ & Very Poor & 0 & 4 \\
\hline 5 & $>300$ & Unsuitable for domestic suitability & 4 & 4 \\
\hline
\end{tabular}

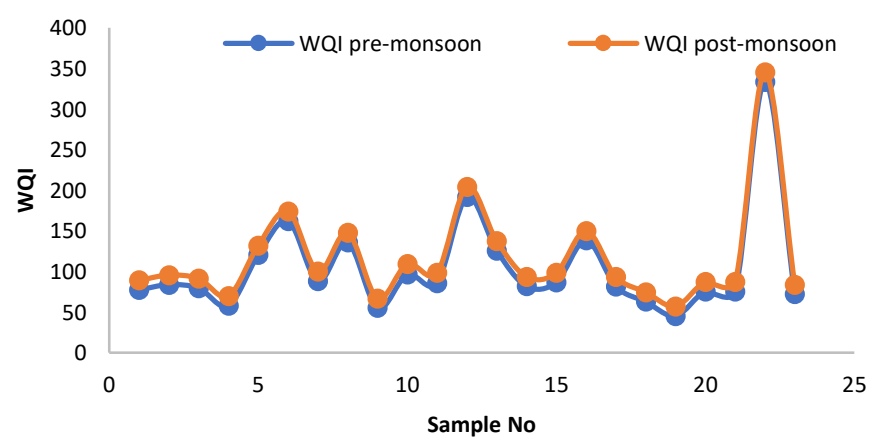

Fig. 3: Plot of Water Quality Index (WQI) Values for Pre-and Post-Monsoon Season. 
Table 8: Computation of Water Quality Index for Groundwater Samples

\begin{tabular}{|c|c|c|c|c|}
\hline Sample No. & WQI pre-monsoon & Water type & WQI Post-monsoon & Water type \\
\hline 1 & 77.33 & Good & 89.07 & Good \\
\hline 2 & 83.69 & Good & 95.43 & Good \\
\hline 3 & 79.53 & Good & 91.27 & Good \\
\hline 4 & 58.13 & Good & 69.87 & Good \\
\hline 5 & 120.31 & Poor & 132.05 & Poor \\
\hline 6 & 162.28 & Poor & 174.02 & Poor \\
\hline 7 & 88.14 & Good & 99.88 & Good \\
\hline 8 & 135.91 & Poor & 147.65 & Poor \\
\hline 9 & 55.15 & Good & 66.89 & Good \\
\hline 10 & 96.35 & Good & 108.09 & Poor \\
\hline 11 & 85.74 & Good & 97.48 & Good \\
\hline 12 & 191.60 & Poor & 203.34 & Very Poor \\
\hline 13 & 125.74 & Poor & 137.48 & Poor \\
\hline 14 & 81.80 & Good & 93.54 & Good \\
\hline 15 & 86.67 & Good & 98.41 & Good \\
\hline 16 & 138.25 & Poor & 149.99 & Poor \\
\hline 17 & 81.60 & Good & 93.34 & Good \\
\hline 18 & 62.83 & Good & 74.57 & Good \\
\hline 19 & 45.37 & Excellent & 57.11 & Good \\
\hline 20 & 75.51 & Good & 87.25 & Good \\
\hline 21 & 75.45 & Good & 87.19 & Good \\
\hline 22 & 333.55 & Unsuitable & 345.29 & Unsuitable \\
\hline 23 & 72.09 & Good & 83.83 & Good \\
\hline
\end{tabular}

\section{Water quality index (WQI) for domestic suitability}

Quality of water is very significant to human because it has a direct link with human health and welfare (Chatterjee \& Raziuddin, 2002; Srivastava, et al, 2007; Shaikh, et al, 2020). The water quality index (WQI) is an important tool to determine the drinking water quality in urban, rural and industrial area. Groundwater samples $(n=23)$ and its WQI values as well as its types are presented in Table 7 and fig .3. Thus, the WQI can be classified as excellent water type, if the WQI values are less than 50; good water type, if the WQI values ranged from 50 to 100; poor water type, if the WQI values ranged between 100 and 200; very poor water type, if the WQI values ranged from 200 to 300, and unsuitable for drinking water type, if the WQI values larger than 300. The WQI value and water type of the individual samples are presented in Table 8. The WQI ranges from 45.37 to 333.55 and 57.11 to 345.29 with an average of 104.91 and 116.65 for pre- and post-monsoon seasons respectively. During pre-monsoon, $4 \%$ ( 1 sample) of groundwater samples were excellent; $65 \%$ ( 15 samples) were good; $26 \%$ (6 samples) were poor; and 4\% (1 sample) were unsuitable for domestic suitability. In post-monsoon, $65 \%$ (15 samples) of water samples were good; $26 \%$ (6 samples) were poor; $4 \%$ (1 sample) were very poor and $4 \%$ ( 1 sample) were unsuitable for domestic suitability (Table 6). Quality rating of the water is established from WQI as it is established from different physicochemical parameters in different seasons of the groundwater body. It is confirmed that majority groundwater samples from both seasons are good for drinking purpose as well as domestic suitability. At certain place poor quality of groundwater is mainly due to inputs from agricultural discharge.

\section{Conclusion}

This study is focussed on understanding the geochemistry of groundwater, its seasonal variation and degree of pollution. The study indicating that groundwater is slightly alkaline type. Electrical conductivity shows that $22 \%$ samples were exceeded the permissible limit, during pre- and post-monsoon season. The TDS of ground water sample in the post-monsoon season was higher as compared to the premonsoon season which may be attributed due to leaching of salts from ground surface by recharge. Analysed physicochemical parameters like turbidity, total hardness, calcium, magnesium, sodium, potassium, total alkinity, chloride, sulphate is mostly in within limits. Nitrate ion content exceeds the recommended limit of drinking in many groundwater samples. Nitrate of groundwater samples shows that $39 \%$ and $57 \%$ samples were exceeding the permissible limit in pre- and post-monsoon season and the origin of $\mathrm{NO}_{3}$ is derived from agricultural areas due to leaching process from plant nutrient and nitrate fertilizers. Fluoride is higher during pre-monsoon season $(1.92 \mathrm{mg} / \mathrm{L}) \mathrm{due}$ to leaching from fluoride rich rocks and easier accessibility of rain water to weathered rock, long-term irrigation processes, semi-arid climate and long residence time of groundwater. Based on water quality index, it was inferred that $4 \%$ of groundwater sample is found unfit for drinking purpose in pre- and post-monsoon season. The precautionary measures should be adopted in advance, as well as by reducing the over exploitation of groundwater; construction and proper maintenance of rainwater harvesting structures for recharge will be helpful in preserving, maintaining and improving the groundwater quality.

\section{Acknowledgement}

The authors are extremely grateful to Authority, Groundwater Surveys and Development Agency, for the Support and Encouragement to the author during the course of this work.

\section{References}

[1] Adimalla N, Wu J, (2019). Groundwater quality and associated health risks in a semi-arid region of south India: implication to sustainable groundwater management. Hum Ecol Risk Assess 25(1-2):191-216. https://doi.org/10.1080/10807039.2018.1546550.

[2] Aher, K. R. (2012). Geochemistry and assessment of ground water quality for drinking and irrigation purposes: a case study of Sukhana river subbasin, district Aurangabad, Maharashtra, India. International Journal of Recent Trends in Science and Technology, 4(1), 45-49.

[3] Aher, K. R. (2017). Delineation of groundwater quality for drinking and irrigation purposes: a case study of Bori Nala watershed, district Aurangabad, Maharashtra, India. Journal of Applied Geochemistry, 19(3), 321-338. 
[4] Aher, K. R., \& Deshpande, S. M. (2014). Groundwater Hydrogeochemistry of Mula river Basin. Maharashtra, India, Gondwana Geological Magazine, Spl, 14(167), 716 .

[5] Aher, K. R., \& Dhumal, M. L. (2017). Multivariate Statistical Analyses of Physicochemical Parameters in Groundwater Samples of Borinala Watershed of Aurangabad District, Maharashtra, India. Bulletin of Pure \& Applied Sciences-Geology, 36(1), 18-26. https://doi.org/10.5958/23203234.2017.00002.6.

[6] APHA, (2017). Standard Methods for the Examination of Water and Waste Water, 23 ${ }^{\text {rd }}$ edition, American Public Health Association, Washington DC.

[7] Avvannavar SM, Shrihari S, (2008). Evaluation of water quality index for drinking purposes for river Netravathi, Mangalore, South India. Environ Monit Assess 143(1-3):279-290. https://doi.org/10.1007/s10661-007-9977-7.

[8] Ballukraya, P. N., \& Ravi, R. (1999). Characterisation of groundwater in the unconfined aquifers of Chennai city, India Part 1-Hydrogeochemistry. Journal of the Geological Society of India, 54(1), 1-11.

[9] Bartarya S. K.(1993). Hydrochemistry and rock weathering in a subtropical lesser Himalayan river basin in Kumaun, India. J Hydrol 146:149-174 https://doi.org/10.1016/0022-1694(93)90274-D.

[10] Bauder, T. A., Waskom, R. M., Sutherland, P. L., \& Davis, J. G. (2011). Irrigation water quality criteria. Fact sheet (Colorado State University. Extension). Crop series; no. 0.506.

[11] BIS, (2012). Standards for Water for Drinking and Other Purposes, Bureau of Indian Standards, New Delhi.

[12] Brown, R.M., McCleiland, N.J., Deininger, R.A. and O’Connor, M.F. (1972). A water Quality Index-Crossing the psychological barrier. Jenkis, S.H. (ed.) Proc. Int. Conf.on Water Poll. Res., Jerusalem, 6: 187-197.

[13] Camacho Suarez V.V, Saraiva Okello AML, Wenninger J.W, Uhlenbrook S. (2015). Understanding runoff processes in a semi-arid environment through isotope and hydrochemical hydrograph separations. Hydrol Earth Syst Sci 19(10):4183-4199. https://doi.org/10.5194/hess-19-4183-2015.

[14] Chatterjee, C. and Raziuddin, M. (2002). Determination of water quality index (WQI) of a degraded river in Asansol Industrial area, P.O. Raniganj, District Burdwan, West Bengal. Nature Environment and Pollution Technology, 1(2): 181-189.

[15] Chatterjee, P. R., \& Raziuddin, M. (2007). Studies on the water quality of a water body at Asansol town, West Bengal. Nature, Environment and Pollution Technology, 6(2), 289-292.

[16] Datta, P. S., Deb, D. L., \& Tyagi, S. K. (1996). Stable isotope (180) Investigations on the processes controlling fluoride contamination of groundwater. Journal of Contamination Hydrology, 24, 85-96. https://doi.org/10.1016/0169-7722(96)00004-6.

[17] Deshpande, S. M., Aher, K. R., \& Bhatpude, A. A. (2013). Heavy metal concentrations in ground waters of Chikalthana Area of Aurangabad, India. Journal of Applied Geochemistry, 15(2), 201-212.

[18] Deshpande, S. M., Aher, K. R., \& Gaikwad, G. D. (2012). Assessment of Groundwater Quality and its Suitability for Drinking Uses in Warora tehsil, District Chandrapur, India. Int. J. Rec. Tren. Sci. Technol, 4(3), 2249-8109.

[19] Deshpande, S. M., and Aher, K. R. (2012a). Evaluation of groundwater quality and its suitability for drinking and agriculture use in parts of Vaijapur, District Aurangabad, MS, India. Research Journal of Chemical Sciences, Vol. 2(1),25-31.

[20] Domenico P.A, Schwartz F.W, (1990). Physical and chemical hydrogeology. Wiley, New York.

[21] Freeze, R. A. and J.A. Cherry. (1979). Groundwater. Prentive-hall, Englewood cliffs, NJ, 604p.

[22] Gaikwad H, Shaikh H, Umrikar B, (2018). Evaluation of groundwater quality for domestic and irrigation suitability from Upper Bhima Basin, Western India: a hydro-geochemical perspective. Hydrospatial Anal 2(2):113-123. https://doi.org/10.21523/gcj3.18020204.

[23] Gaikwad G.D., Deshpande S.M., Aher K. R., \& Dasarwar V. R. (2019). Hydrogeochemical Investigation and Qualitative Assessment in Jogeshwari Industrial Area of Aurangabad, Maharashtra, India. Bulletin of Pure \& Applied Sciences-Geology, 38(1), 89-103. https://doi.org/10.5958/23203234.2019.00006.4.

[24] GSDA, (2019). Watershed of Aurangabad region, unpublished document, Deputy Director, Groundwater Surveys and Development Agency, Aurangabad, $2 \mathrm{p}$.

[25] Gupta R, Misra A.K (2018). Groundwater quality analysis of quaternary aquifers in Jhajjar District, Haryana, India: focus on groundwater fluoride and health implications. Alexandria Eng J 57:375-381. https://doi.org/10.1016/j.aej.2016.08.031.

[26] Hem, J. D. (1991). Study and interpretation of the chemical characteristics of natural waters (3rd Ed., Book 2254). Jodhpur: Scientific.

[27] ICMR (1975). Manual of Standards of Quality for Drinking Water Supplies. Indian Council of Medical Research, New Delhi.

[28] Jacks G. (1973). Chemistry of groundwater in a district in Southern India. J Hydrol 18:185-200. https://doi.org/10.1016/0022-1694(73)90047-4

[29] Kadam A.K, Umrikar B.N, Sankhua R.N, (2020). Assessment of recharge potential zones for groundwater development and management using geospatial and MCDA technologies in semiarid region of Western India. SN Appl Sci 2(2):312. https://doi.org/10.1007/s42452-020-2079-7.

[30] Kate S, Kumbhar S, Jamale P, (2020). Water quality analysis of Urun-Islampur City, Maharashtra, India. Appl Water Sci 10(4):1-8. https://doi.org/10.1007/s13201-020-1178-3.

[31] Kumar V.A, Mondal N.C, Ahmed S, (2020). Identification of groundwater potential zones using RS, GIS and AHP techniques: a case study in a part of Deccan Volcanic Province (DVP), Maharashtra, India. J Indian Soc Remote Sens 48:497-511. https://doi.org/10.1007/s12524-019-01086-3.

[32] Kurian, J, (2001). An integrated approach for management of total dissolved solids in reactive

[33] Li P, He X, Guo W, (2019). Spatial groundwater quality and potential health risks due to nitrate ingestion through drinking water: a case study in Yan'an City on the Loess Plateau of northwest China. Hum Ecol Risk Assess Int J 25(1-2):11-31. https://doi.org/10.1080/10807039.2018.1553612.

[34] Madison, R. J., \& Brunett, J. O. (1984). Overview of the occurrence of nitrate in ground water of the United States. In National Water Summary 1984. U.S. Geological Survey, Water Supply Paper, 2275.

[35] Marghade D, (2020). Detailed geochemical assessment \& indexing of shallow groundwater resources in metropolitan city of Nagpur (western Maharashtra, India) with potential health risk assessment of nitrate enriched groundwater for sustainable development. Geochemistry. https://doi.org/10.1016/j.chemer.2020.125627.

[36] Marghade, D., Malpe, D. B., Duraisamy, K., Patil, P. D., \& Li, P. (2020). Hydrogeochemical evaluation, suitability, and health risk assessment of groundwater in the watershed of Godavari basin, Maharashtra, Central India. Environmental Science and Pollution Research, 1-24. https://doi.org/10.1007/s11356-020-10032-7.

[37] Nickson, R. T., McArthur, J. M., Shrestha, B., Kyaw-Nyint, T.O., Lowry, D, (2005). Arsenic and other drinking water quality issues, Muzaffargarh District. Pakistan Applied Geochemistry, 55-68. https://doi.org/10.1016/j.apgeochem.2004.06.004.

[38] Pondhe, G. M., Pawar, N. D., Patil, S. F., \& Dhembare, A. J. (1997). Impact of Sugar Mill Effluent on the Quality of Groundwater. Pollution Research, $16,191-195$.

[39] Purushtotham, D., Rao, A.N., Ravi Prakash, M., Shakeel Ahmed and G. Ashok Babu, (2011). Environmental impact on groundwater of Maheshwaram Watershed, Ranga Reddy district, Andhra Pradesh. J Geol Soc India 77, 539-548. https://doi.org/10.1007/s12594-011-0053-0.

[40] Ramakrishnalah CR, Sadas Hivalah C, Ranganna G, (2009). Assessment of water quality index for the groundwater in Tumkur Taluk, Karnataka state, India. E-J Chem 6(2):523-530. https://doi.org/10.1155/2009/757424.

[41] Ramesh.K and Elango,L.(2011). Groundwater quality and its suitability for domestic and agricultural use in Tondiar river basin, Tamil Nadu, India, Environ Monit Assess https://doi.org/10.1007/s10661-011-2231-3.

[42] Rao, N. S., \& Rao, P. S. (2010). Major ion chemistry of groundwater in a river basin: a study from India. Environmental Earth Sciences, 61(4), 757775. https://doi.org/10.1007/s12665-009-0389-6.

[43] Robinson, W. D., \& Edington, G. (1946). Fluorine in soils. Soil Science, 61, 341-353. https://doi.org/10.1097/00010694-194605000-00001.

[44] Salve P. L., \& Aher K. R. (2016). Groundwater Quality studies of Selected Sources in Vaijapur Block, District Aurangabad (MS). Journal of current Medical and Applied sciences, 9(2), 65-68.

[45] Sawyer, C. N., \& McCarty, P. L. (1967). Chemistry for sanitary engineers. McGraw-Hill, New York. 
[46] Shaikh, H., Gaikwad, H., Kadam, A., \& Umrikar, B. (2020). Hydrogeochemical characterization of groundwater from semiarid region of western India for drinking and agricultural purposes with special reference to water quality index and potential health risks assessment. Applied Water Science, 10(9), 1-16. https://doi.org/10.1007/s13201-020-01287-z.

[47] Sharma D, Kansal A, (2011). Water quality analysis of River Yamuna using water quality index in the national capital territory, India (2000-2009). Appl Water Sci 1(3-4):147-157. https://doi.org/10.1007/s13201-011-0011-4.

[48] Singh, K.P. and Parwana, H. K. (1992). Ground water pollution due to industrial waste water in Punjab state and strategies, I.J. Environmental Protection, 19 (4).

[49] Srinivasamoorthy K., Vasanthavigar M., Chidambaram S., Anandhan P. and Sarma V. S. (2011). Characterisation of groundwater chemistry in an Eastern coastal area of Cuddalore District, Tamil Nadu, Jour. Geol. Soc. India, Vol. 78: 549-558. https://doi.org/10.1007/s12594-011-0122-4.

[50] Srinivasamoorthy, K., Chidambaram, S., \& Vasanthavigar, M. (2008). Geochemistry of fluorides in groundwater: Salem District, Tamilnadu, India. Journal of Environnemental Hydrology, 1, 16-25.

[51] Srinivasamoorthy, K., Vijayaraghavan, K., Vasanthavigar, M., Sarma, S., Chidambaram, S., Anandhan, P., \& Manivannan, R. (2012). Assessment of groundwater quality with special emphasis on fluoride contamination in crystalline bed rock aquifers of Mettur region, Tamilnadu, India. Arabian Journal of Geosciences, 5(1), 83-94. https://doi.org/10.1007/s12517-010-0162-x.

[52] Srivastava A.K, Parimal P.S, (2020). Source rock weathering and groundwater suitability for irrigation in Purna alluvial basin, Maharashtra, central India. J Earth Syst Sci 129(1):52. https://doi.org/10.1007/s12040-019-1312-5.

[53] Srivastava, A. K., Mishra, D. K., Tripathi, S., \& Singh, P. (2007). Determination of water quality index and suitability of ground water in a college in Balrampur, U. P. Nature, Environment and Pollution Technology, 6(2), 315-319.

[54] Trivedi, R.K. and Goel,P.K.,(1984). Chemical and biological methods for water pollution studies. Environmental Publications Karad, India 215.

[55] U.S. Salinity Lab. Staff. (1954). Diagnosis and improvement of saline and alkali soils. U.S. Dept. Agri. Hand book-60, 160 p.

[56] Vasanthavigar M, Srinivasamoorthy K, Vijayaragavan K, Ganthi RR, Chidambaram S, Anandhan P, Manivannan R, Vasudevan S, (2010). Application of water quality index for groundwater quality assessment: Thirumanimuttar sub-basin, Tamilnadu, India. Environ Monit Assess 171(1-4):595609. https://doi.org/10.1007/s10661-009-1302-1.

[57] Vasanthavigar M., Srinivasamoorthy K., \& Prasanna M.V. (2012). Evaluation of groundwater suitability for domestic, irrigational, and industrial purposes: a case study from Thirumanimuttar river basin, Tamilnadu, India. Environmental Monitoring and Assessment, 184(1), 405-420. https://doi.org/10.1007/s10661-011-1977-y.

[58] Verma A, Yadav B.K, Singh N.B, (2020). Hydrochemical monitoring of groundwater quality for drinking and irrigation use in Rapti Basin. SN Appl Sci 2(3):1-15. https://doi.org/10.1007/s42452-020-2267-5.

[59] Wagh, V., Panaskar, D., Aamalawar, M., Lolage, Y., Mukate, S., \& Adimalla, N. (2018). Hydro-chemical characterisation and groundwater suitability for drinking and irrigation uses in semiarid region of Nashik, Maharashtra, India. Hydrospatial Anal 2:43-60. https://doi.org/10.21523/gcj3.18020104.

[60] Whittemore, D. O., McGregor, K. M., \& Marotz, G. A. (1989). Effects of variations in recharge on groundwater quality. Journal of hydrology, 106(12), 131-145. https://doi.org/10.1016/0022-1694(89)90170-4.

[61] WHO (2011). International Standards for Drinking Water. World Health Organization, Geneva, Switzerland.

[62] Wu J, Sun Z, (2016). Evaluation of shallow groundwater contamination and associated human health risk in an alluvial plain impacted by agricultural and industrial activities, mid-west China. Expo Health 8:311-329. https://doi.org/10.1007/s12403-015-0170-x. 